\title{
Changes in foot progression angle during gait reduce the knee adduction moment and do not increase hip moments in individuals with knee osteoarthritis
}

Kirsten Seagers ${ }^{1}$, Scott D. Uhlrich ${ }^{1,2,3}$, Julie A. Kolesar ${ }^{2,3}$, Madeleine Berkson ${ }^{2}$, Janelle M. Kaneda $^{3}$, Gary S. Beaupre ${ }^{1,2}$, Scott L. Delp ${ }^{1,3,4}$

${ }^{1}$ Department of Mechanical Engineering, Stanford University, Stanford, California, United States

${ }^{2}$ Department of Veterans Affairs Healthcare System, Palo Alto, California, United States

${ }^{3}$ Department of Bioengineering, Stanford University, Stanford, California, United States

${ }^{4}$ Department of Orthopaedic Surgery, Stanford University, Stanford, California, United States 


\begin{abstract}
People with knee osteoarthritis who adopt a modified foot progression angle (FPA) during gait often benefit from a reduction in the knee adduction moment and knee pain. It is unknown, however, whether changes in the FPA increase hip moments, a surrogate measure of hip loading, which may increase the risk of hip pain or osteoarthritis. This study examined how altering the FPA affects hip moments. Individuals with knee osteoarthritis walked on an instrumented treadmill with their baseline gait, $10^{\circ}$ toe-in gait, and $10^{\circ}$ toe-out gait. A musculoskeletal modeling package was used to compute joint moments from the experimental data. Fifty participants were selected from a larger study who reduced their peak knee adduction moment with a modified FPA. In this group, participants reduced the first peak of the knee adduction moment by $7.6 \%$ with $10^{\circ}$ toe-in gait and reduced the second peak by $11.0 \%$ with $10^{\circ}$ toe-out gait. Modifying the FPA reduced the early-stance hip abduction moment, at the time of peak hip contact force, by $4.3 \% \pm 1.3 \%$ for $10^{\circ}$ toe-in gait $(\mathrm{p}=0.005)$ and by $4.6 \% \pm 1.1 \%$ for $10^{\circ}$ toe-out gait $(\mathrm{p}<0.001)$ without increasing the flexion and internal rotation moments $(\mathrm{p}>0.15)$. In summary, when adopting a FPA modification that reduced the knee adduction moment, participants did not increase surrogate measures of hip loading.
\end{abstract}

Keywords: gait modifications, non-surgical intervention, joint loading, musculoskeletal modeling, osteoarthritis 


\section{Introduction}

Of the 30 million Americans who suffer from osteoarthritis (OA) (Cisternas et al., 2016), an estimated 14 million have symptomatic knee OA (Deshpande et al., 2016). People with knee OA have a 25\% lifetime risk of developing hip OA (Murphy et al., 2010) due to shared risk factors, which place multiple joints at risk for OA (Felson, 2004; Issa et al., 2012). Obesity, one of these risk factors, increases compressive loading in both the knee (Messier et al., 2014) and the hip (Lerner and Browning, 2016). These joint forces influence OA initiation and progression (Andriacchi et al., 2004; Felson, 2013). Since direct measurement of compressive joint forces in a native joint is not possible, joint moments are often used as surrogate measures of joint loading.

The knee adduction moment (KAM) is correlated with knee OA presence (Baliunas et al., 2002), knee pain (Robbins et al., 2011; Thorp et al., 2007), knee cartilage degradation (Chehab et al., 2014), and predicts knee OA progression (Miyazaki, 2002). People can change their KAM by modifying features of their gait, such as trunk sway or walking speed (Hunt et al., 2008;

Mündermann et al., 2004). Gait modifications are attractive because they can potentially reduce pain without surgery or external devices. One such gait modification is changing the foot progression angle (FPA), i.e., toeing-in or toeing-out. Altering the FPA requires changes to lower-body kinematics (Shull et al., 2013) and muscle coordination (Charlton et al., 2018; Rutherford et al., 2010). Toeing-in generally reduces the first peak of the KAM (Shull et al., 2013), whereas toeing-out reduces the second peak of the KAM (Guo et al., 2007). With personalized FPA modifications, the number of individuals who can reduce their peak KAM is maximized (Uhlrich et al., 2018). Studies that evaluate the efficacy of KAM-targeted gait modifications focus primarily on the effects at the knee, although Mündermann et al. (2005) suggested the need to evaluate the effects of these modifications on other joints. 
As research on FPA modifications for people with knee OA progresses towards a deployable intervention (Hunt et al., 2018; Xia et al., 2020), it is important to understand their effects more comprehensively, particularly at the hip. Increased hip moments alter the compressive loading environment in the joint (Simic et al., 2011) and are associated with hip OA symptoms (Hall et al., 2019), presence (Maly et al., 2013), severity (Diamond et al., 2018), and progression (Tateuchi et al., 2017). Some studies have examined the effect of gait modifications on hip contact forces, kinetics, and kinematics to minimize torsional loading and loosening of total hip replacements (Bowsher and Vaughan, 1995) and to reduce hip contact forces for patients with hip pathology (Wesseling et al., 2015). However, gait modifications that target knee loading have not been investigated for their effect on the hip. Therefore, an important next step is to understand how interventions that target knee loading affect hip kinetics, kinematics, and spatiotemporal parameters of gait.

This study analyzes the biomechanical effects of a FPA modification on the hip in individuals with medial compartment knee OA. The purpose of this research is twofold: (1) determine if FPA modifications increase peak hip moments and (2) understand the lowerextremity kinetic, kinematic, and spatiotemporal parameter changes that occur when modifying the FPA. Our results provide insight into whether gait modifications for knee osteoarthritis could have unintended deleterious effects at the hip.

\section{Methods}

\section{Data Collection}

Participant data were analyzed from a clinical study (clinicaltrials.gov NCT02767570) that trained people with medial compartment knee OA to walk with a modified FPA to reduce 
their peak KAM. One hundred seven people provided informed consent in agreement with a Stanford University Institutional Review Board approved protocol. Inclusion criteria included medial compartment knee OA grade between one and three on the Kellgren-Lawrence scale from anterior-posterior weightbearing radiographs; medial knee pain of three or greater on an 11-point numeric rating scale; the ability to walk safely on a treadmill without assistance for 25 minutes; and BMI less than 35. The limb examined for each participant was chosen based on the presence of radiographic and symptomatic medial knee OA. If medial knee OA was present in both knees, the more symptomatic side was analyzed.

The data collection methods have been detailed previously (Uhlrich et al., 2020) and are outlined here. Experimental gait data were collected during a visit where different FPA modifications were evaluated for their effect on KAM peaks (Uhlrich et al., 2020). First, a standing calibration trial was performed, followed by a functional hip joint center calibration trial during which participants were asked to circumduct their hips (Piazza et al., 2004). Participants then walked naturally at a self-selected speed on an instrumented split belt treadmill (Bertec Corporation, Columbus, OH, USA) while ground reaction force data and optical marker positions were recorded (Motion Analysis Corporation, Santa Rosa, CA, USA). Vibrotactile biofeedback (Engineering Acoustics, Casselberry, FL, USA) was given to teach participants to toe-in and toe-out by $10^{\circ}$ relative to their natural FPA. The absolute FPA was calculated as the angle between the line connecting the calcaneus and second metatarsal head marker positions and the forward direction of the treadmill during 15-40\% of stance (Rutherford et al., 2008).

Of the one hundred seven participants who completed this task in the clinical study, fifty were included in this analysis (Table 1). Selection for this analysis required participants to reduce the first or second peak KAM by at least $5 \%$ with either a $10^{\circ}$ toe-in or a $10^{\circ}$ toe-out gait 
modification as compared to baseline. The first and second peak of the KAM were defined as the maximum values during $0-50 \%$ and $51-100 \%$ of the stance phase, respectively. Participants were excluded from this analysis if they used the treadmill handrails during walking trials or had greater than an estimated $1 \mathrm{~cm}$ of soft tissue between the anterior superior iliac spine marker and the anatomical bony landmark.

\section{Musculoskeletal Modeling}

A multicompartment knee model (Lerner et al., 2015) with adduction and flexion degrees of freedom was incorporated into a full-body musculoskeletal model with 25 degrees of freedom and 80 muscle-tendon units (Rajagopal et al., 2016). The arms were removed from the model and the metatarsophalangeal joints were locked.

Scaling the generic musculoskeletal model to match the dimensions of each subject was a two-step process. First, long-leg radiograph measurements were used to adjust frontal plane tibiofemoral alignment and pelvic width. The frontal plane leg alignment was found by measuring the angle (ImageJ, National Institute of Mental Health, Bethesda, Maryland, USA) between a line connecting the hip and knee joint centers and a line connecting the knee and ankle joint centers (Lerner et al., 2015). The pelvic width was defined as the distance between the hip joint centers identified on radiograph. Second, the remainder of the model bodies were scaled using locations of joint centers and anatomical markers in OpenSim 4.0 (Seth et al., 2019).

Inverse kinematics was performed to compute hip adduction, flexion, and internal rotation angles. Results were filtered with a $4^{\text {th }}$ order Butterworth filter and cutoff frequency of 15Hz. These kinematics and ground reaction forces, also filtered at $15 \mathrm{~Hz}$, were used in inverse dynamics to calculate external hip abduction, extension, and internal rotation moments.

\section{Data and Statistical Analysis}


For each gait trial (baseline, $10^{\circ}$ toe-in, and $10^{\circ}$ toe-out), the last 20 steps that achieved the target FPA, within $2.5^{\circ}$, were analyzed. Each biomechanical metric of interest was calculated for every step and averaged over each walking condition.

Hip moments were normalized by participant mass and compared between walking conditions for two primary kinetic metrics: the early-stance hip moment and angular impulse. To estimate the hip moment at the time of greatest hip loading, the early-stance hip moment was computed as the average moment during $15-20 \%$ of stance phase, the time of first peak hip contact force (Bergmann et al., 2001; Wesseling et al., 2015). For analysis during the entire stance phase, the angular impulse was computed as the area under the moment absolute value curve during stance phase. The sum of the angular impulse in the frontal, sagittal, and transverse planes defined the cumulative angular impulse. For kinematic analysis, joint angles were averaged during stance and compared between walking conditions to capture global changes. Peak angles were also compared between conditions. Spatiotemporal parameters were also determined for comparison, including FPA, stride length, and step width. Stride length was defined as the difference in right and left foot calcaneus marker positions at time of heel strike. Step width was defined as the distance between the right and left foot center of pressure at $50 \%$ of stance (Donelan et al., 2001).

Statistical comparisons of $10^{\circ}$ toe-in and $10^{\circ}$ toe-out gait metrics compared to baseline were performed in MATLAB R2020a (MathWorks Corporation, Natick, MA USA) using a Wilcoxon signed-rank test $(\alpha=0.05)$. Bonferroni corrected $\mathrm{p}$-values for multiple comparisons across FPA conditions were reported to mitigate type I error.

\section{Results}


Participants adopted an FPA modification that reduced the peak knee adduction moment and did not increase surrogate measures of hip loading. On average, $10^{\circ}$ toe-in modifications reduced the first peak of the KAM by $7.6 \%$ compared to baseline, and $10^{\circ}$ toe-out reduced the second peak by $11.0 \%$. The early-stance hip abduction moment was significantly lower during $10^{\circ}$ toe-in gait $(-4.3 \% \pm 1.3 \% ; \mathrm{p}=0.005)$ and during $10^{\circ}$ toe-out gait $(-4.6 \% \pm 1.1 \% ; \mathrm{p}<0.001)$ compared to baseline. There were no significant differences $(\mathrm{p}>0.05)$ in the early-stance hip extension moment or early-stance internal rotation moment for $10^{\circ}$ toe-in or toe-out conditions compared to baseline (Figure 1, Figure 2).

The frontal plane angular impulse was significantly reduced compared to baseline for $10^{\circ}$ toe-in gait $(-2.3 \% \pm 0.7 \%, \mathrm{p}<0.01)$ and for $10^{\circ}$ toe-out gait $(-6.9 \% \pm 0.8 \%, \mathrm{p}<0.001)$, but no significant differences $(\mathrm{p}>0.05)$ were observed in the sagittal or transverse plane (Figure 2). There was no difference in the cumulative angular impulse for $10^{\circ}$ toe-in gait compared to baseline; however, the $10^{\circ}$ toe-out cumulative angular impulse was significantly lower $(-3.6 \% \pm$ $0.7 \%, \mathrm{p}<0.001)$. While on average there were no increases in the cumulative angular impulse, some individuals experienced increases. The largest observed cumulative angular impulse increase from baseline was $7.7 \%$ and $9.2 \%$ for $10^{\circ}$ toe-in and $10^{\circ}$ toe-out gait, respectively.

Changes in hip kinematics were minimal. Participants walked with a decreased average hip adduction angle for $10^{\circ}$ toe-in gait $\left(-0.4^{\circ} \pm 1.1^{\circ}, \mathrm{p}=0.045\right)$ and $10^{\circ}$ toe-out gait $\left(-1.6^{\circ} \pm 1.2^{\circ}\right.$, $\mathrm{p}<0.001$ ) compared to baseline (Figure 4). Peak hip adduction angles also decreased for both toein $\left(-0.7^{\circ} \pm 1.5^{\circ}, \mathrm{p}=0.009\right)$ and toe-out $\left(-1.8^{\circ} \pm 1.5^{\circ}, \mathrm{p}=0.005\right)$ gait compared to baseline. Participants walked with increased average hip flexion when toeing-in $\left(2.7^{\circ} \pm 2.2^{\circ}, \mathrm{p}<0.001\right)$ and toeing-out $\left(1.7^{\circ} \pm 2.1^{\circ}, \mathrm{p}<0.001\right)$. Both modifications had a lower peak extension angle compared to baseline (toe-in: $-3.2^{\circ} \pm 2.7^{\circ}, \mathrm{p}<0.001$; toe-out: $-2.5^{\circ} \pm 2.7^{\circ}, \mathrm{p}<0.001$ ). The average 
hip internal rotation angle increased by an average of $4.4^{\circ} \pm 2.6^{\circ}(\mathrm{p}<0.001)$ when walking with a $10^{\circ}$ toe-in gait and decreased by $5.7^{\circ} \pm 1.3^{\circ}(\mathrm{p}<0.001)$ with a $10^{\circ}$ toe-out gait. Similar directional changes were seen in the peak hip internal rotation angle compared to baseline (toe-in: $4.6^{\circ} \pm$ $2.7^{\circ}, \mathrm{p}<0.001$; toe-out: $\left.-5.8^{\circ} \pm 1.6^{\circ}, \mathrm{p}<0.001\right)$. Changes in spatiotemporal parameters were also observed. Stride length decreased with both FPA modifications $(\mathrm{p}<0.001)$, while step width increased only for $10^{\circ}$ toe-out gait $(\mathrm{p}<0.001)$ (Table 2$)$.

\section{Discussion}

We analyzed how changing the FPA during walking affects hip moments in fifty individuals with medial knee OA. Both $10^{\circ}$ toe-in and $10^{\circ}$ toe-out modifications decreased the early-stance hip abduction moment and the frontal plane angular impulse. On average, adopting an FPA modification, that reduced the KAM, did not significantly increase the hip moments at time of peak hip contact force, the angular impulse, or the cumulative angular impulse. This suggests that this intervention may not increase hip loading and place the hip joint at increased risk of OA in most participants. However, some participants did increase their cumulative angular impulse moment with the FPA modification, which could be detrimental to hip joint health. For people with hip joint pathology, or who are at high risk of developing hip OA, the effect of a FPA gait modification at the hip should be evaluated individually.

Our observed decrease in the hip abduction moment is consistent with other studies. The $4 \%$ decrease in the early-stance hip abduction moment when toeing-in and toeing-out was smaller than the $16 \%$ decrease observed at the time of peak KAM in a healthy population (Kettlety et al., 2018), which could be due to the varying participant population and period of the gait cycle when the moments were analyzed. Similarly, the reductions in early-stance hip 
abduction moment $\left(10^{\circ}\right.$ toe-in: $0.03 \mathrm{Nm} / \mathrm{kg} ; 10^{\circ}$ toe-out: $\left.0.04 \mathrm{Nm} / \mathrm{kg}\right)$ agreed directionally with the $0.1 \mathrm{Nm} / \mathrm{kg}$ decrease Wesseling et al., (2015) reported from simulated perturbations of frontal plane hip kinematics. The magnitude differences are unsurprising when comparing simulation predictions to experimental results.

Kinematic and spatiotemporal changes were also observed when participants modified their FPA. Changes in the hip rotation angle were less than the change in FPA, which aligns with results from Cibulka et al., (2016), who found that FPA modifications elicit changes at multiple lower-extremity joints. In addition to the rotational kinematics changes, the average hip adduction angle decrease relates to the increase in step width for toe-out gait. Other studies (Kettlety et al., 2020; Uhlrich et al., 2018) have also observed that step width increases with a modified FPA resulting from less hip adduction. While the decrease in hip adduction angle and increase in step width are small, these changes increase medio-lateral stability (Meyer et al., 2018). Additionally, participants decreased stride length, which can increase double-limb support time, resulting in a more stable gait (Shkuratova et al., 2004). The changes to kinematics and spatiotemporal parameters may attenuate over time as an individual becomes more comfortable with the gait modification.

It is important to identify the limitations of our study. First, we estimated the effect of FPA modifications on surrogate measures of hip loading. Electromyography was not used in this study, but, in the future, validated electromyography-informed simulations could estimate changes in muscle and hip contact forces. The surrogate measure of hip loading that we used, joint moments, is related to muscle and joint contact forces, which provides an estimate of the directional change in hip contact force. Meyer et al. (2018) reported that a decrease in the hip abduction moment and hip adduction angle reduced the work generated by the hip abductor 
muscles and decreased hip loading in people with hip OA. Second, it is challenging to accurately measure axial rotations at lower-extremity joints with optical motion capture (Gorton et al., 2009). Fluoroscopy could be used in future studies to assess the distribution of axial rotations more accurately across lower-extremity joints.

Several studies have shown that foot progression angle modifications can provide meaningful reductions in the knee adduction moment. Our results add to this growing body of evidence and show that foot progression angle modifications reduced frontal plane hip moments without increasing the moments in other planes. This suggests that changes in foot progression angle that aim to reduce knee loading, on average, do not increase surrogate measures of hip loading. Results from this study, and our previous study (Uhlrich et al., 2020), suggest that changes in foot progression angle during gait should be personalized to determine the best intervention for each individual.

\section{Acknowledgements}

We would like to thank the participants involved in the study. This work was supported by the Sang Samuel Wang Stanford Graduate Fellowship, NSF Graduate Research Fellowships (DGE1147470 and DGE-1656518), NIH grant P41EB027060, and by Merit Review Award I01 RX001811 from the United States Department of Veterans Affairs Rehabilitation Research and Development Service. 


\section{References}

Andriacchi, T.P., Mündermann, A., Smith, R.L., Alexander, E.J., Dyrby, C.O., Koo, S., 2004. A Framework for the in Vivo Pathomechanics of Osteoarthritis at the Knee. Annals of Biomedical Engineering 32, 447-457.

Baliunas, A.J., Hurwitz, D.E., Ryals, A.B., Karrar, A., Case, J.P., Block, J.A., Andriacchi, T.P., 2002. Increased knee joint loads during walking are present in subjects with knee osteoarthritis. Osteoarthritis and Cartilage 10, 573-579.

Bergmann, G., Deuretzbacher, G., Heller, M., Graichen, F., Rohlmann, A., Strauss, J., Duda, G.N., 2001. Hip contact forces and gait patterns from routine activities. Journal of Biomechanics 34, 859-871.

Bowsher, K.A., Vaughan, C.L., 1995. Effect of foot-progression angle on hip joint moments during gait. Journal of Biomechanics 28, 759-762.

Charlton, J.M., Hatfield, G.L., Guenette, J.A., Hunt, M.A., 2018 Toe-in and toe-out walking require different lower limb neuromuscular patterns in people with knee osteoarthritis. Journal of Biomechanics 76, 112-118.

Chehab, E.F., Favre, J., Erhart-Hledik, J.C., Andriacchi, T.P., 2014. Baseline knee adduction and flexion moments during walking are both associated with 5-year cartilage changes in patients with medial knee osteoarthritis. Osteoarthritis and Cartilage 22, 1833-1839.

Cibulka, M.T., Winters, K., Kampwerth, T., McAfee, B., Payne, L., Roeckenhaus, T., Ross, S.A., 2016. Predicting Foot Progression Angle during Gait using Two Clinical Measures in Healthy Adults, a Preliminary Study. International Journal of Sports Physical Therapy 11, 400-408.

Cisternas, M.G., Murphy, L., Sacks, J.J., Solomon, D.H., Pasta, D.J., Helmick, C.G., 2016. Alternative Methods for Defining Osteoarthritis and the Impact on Estimating Prevalence in a US Population-Based Survey: OA Prevalence in a Population-Based Survey. Arthritis Care \& Research 68, 574-580.

Deshpande, B.R., Katz, J.N., Solomon, D.H., Yelin, E.H., Hunter, D.J., Messier, S.P., Suter, L.G., Losina, E., 2016. Number of Persons With Symptomatic Knee Osteoarthritis in the US: Impact of Race and Ethnicity, Age, Sex, and Obesity: Symptomatic Knee OA in the US. Arthritis Care \& Research 68, 1743-1750.

Diamond, L.E., Allison, K., Dobson, F., Hall, M., 2018. Hip joint loading during walking in people with hip osteoarthritis: a systematic review and meta-analysis. Osteoarthritis and Cartilage, Abstracts from the 2018 OARSI World Congress on Osteoarthritis 26, S370.

Donelan, J.M., Kram, R., Kuo, A.D., 2001. Mechanical and metabolic determinants of the preferred step width in human walking. Proceedings of the Royal Society: Biological Sciences 268, 1985-1992.

Felson, D.T., 2013. Osteoarthritis as a disease of mechanics. Osteoarthritis and Cartilage 21, 1015.

Felson, D.T., 2004. Risk factors for osteoarthritis: understanding joint vulnerability. Clinical Orthopaedics and Related Research 427, S16-21.

Gorton III, G.E., Hebert, D.A., Gannotti, M.E., 2009. Assessment of the kinematic variability among 12 motion analysis laboratories. Gait \& Posture 29, 398-402.

Guo, M., Axe, M.J., Manal, K., 2007. The influence of foot progression angle on the knee adduction moment during walking and stair climbing in pain free individuals with knee osteoarthritis. Gait \& Posture 26, 436-441. 
Hall, M., Chabra, S., Shakoor, N., Leurgans, S.E., Demirtas, H., Foucher, K.C., 2019. Hip joint moments in symptomatic vs. asymptomatic people with mild radiographic hip osteoarthritis. Journal of Biomechanics 96, 109347.

Hunt, M.A., Birmingham, T.B., Jenkyn, T.R., Giffin, J.R., Jones, I.C., 2008. Measures of frontal plane lower limb alignment obtained from static radiographs and dynamic gait analysis. Gait \& Posture 27, 635-640.

Hunt, M.A., Charlton, J.M., Krowchuk, N.M., Tse, C.T.F, Hatfield, G.L., 2018. Clinical and biomechanical changes following a 4-month toe-out gait modification program for people with medial knee osteoarthritis: a randomized controlled trial. Osteoarthritis and Cartilage 26, 903-911.

Issa, R., Griffin, T., 2012. Pathobiology of obesity and osteoarthritis: integrating biomechanics and inflammation. Pathobiology of Aging \& Age-related Diseases 2, 17470.

Kettlety, S., Lindsey, B., Eddo, O., Prebble, M., Caswell, S., Cortes, N., 2020. Changes in hip mechanics during gait modification to reduce knee adduction moment. Journal of Biomechanics 99, 1-7.

Lerner, Z.F., Browning, R.C., 2016. Compressive and shear hip joint contact forces are affected by pediatric obesity during walking. Journal of Biomechanics 49, 1547-1553.

Lerner, Z.F., DeMers, M.S., Delp, S.L., Browning, R.C., 2015. How tibiofemoral alignment and contact locations affect predictions of medial and lateral tibiofemoral contact forces. Journal of Biomechanics 48, 644-650.

Maly, M.R., Robbins, S.M., Stratford, P.W., Birmingham, T.B., Callaghan, J.P., 2013. Cumulative knee adductor load distinguishes between healthy and osteoarthritic knees-A proof of principle study. Gait \& Posture 37, 397-401.

Manal, K., Gardinier, E., Buchanan, T.S., Snyder-Mackler, L., 2015. A More Informed Evaluation of Medial Compartment Loading: the Combined Use of the Knee Adduction and Flexor Moments. Osteoarthritis and Cartilage 23(7), 1107-1111.

Messier, S.P., Pater, M., Beavers, D.P., Legault, C., Loeser, R.F., Hunter, D.J., DeVita, P., 2014. Influences of alignment and obesity on knee joint loading in osteoarthritic gait. Osteoarthritis and Cartilage 22, 912-917.

Meyer, C., Wesseling, M., Corten, K., Nieuwenhuys, A., Monari, D., Simon, J., Jonkers, I., Desloovere, K., 2018. Hip movement pathomechanics of patients with hip osteoarthritis aim at reducing hip joint loading on the osteoarthritic side. Gait \& Posture, 59, 11-17.

Miyazaki, T., 2002. Dynamic load at baseline can predict radiographic disease progression in medial compartment knee osteoarthritis. Annals of the Rheumatic Diseases 61, 617-622.

Mündermann, A., Dyrby, C.O., Hurwitz, D.E., Sharma, L., Andriacchi, T.P., 2004. Potential strategies to reduce medial compartment loading in patients with knee osteoarthritis of varying severity: Reduced walking speed: Relationship of Walking Speed to Maximum Knee Adduction Moment. Arthritis \& Rheumatism 50, 1172-1178.

Mündermann, A., Dyrby, C.O., Andriacchi, T.P., 2005. Secondary gait changes in patients with medial compartment knee osteoarthritis: Increased load at the ankle, knee, and hip during walking. Arthritis \& Rheumatism 52, 2835-2844.

Murphy, L.B., Helmick, C.G., Schwartz, T.A., Renner, J.B., Tudor, G., Koch, G.G., Dragomir, A.D., Kalsbeek, W.D., Luta, G., Jordan, J.M., 2010. One in four people may develop symptomatic hip osteoarthritis in his or her lifetime. Osteoarthritis and Cartilage 18, 13721379. 
Piazza, S.J., Erdemir, A, Okita, N, Cavanagh, P.R., 2004. Assessment of the functional method of hip joint center location subject to reduced range of hip motion. Journal of Biomechanics 37, 349-356

Rajagopal, A., Dembia, C.L., DeMers, M.S., Delp, D.D., Hicks, J.L., Delp, S.L., 2016. FullBody Musculoskeletal Model for Muscle-Driven Simulation of Human Gait. IEEE Transactions on Biomedical Engineering 63, 2068-2079.

Robbins, S.M., Birmingham, T.B., Callaghan, J.P., Jones, G.R., Chesworth, B.M., Maly, M.R., 2011. Association of pain with frequency and magnitude of knee loading in knee osteoarthritis. Arthritis Care \& Research 63, 991-997.

Rutherford, D.J., Hubley-Kozey, C.L., Deluzio, K.J., Stanish, W.D., Dunbar, M., 2008. Foot progression angle and the knee adduction moment: a cross-sectional investigation in knee osteoarthritis. Osteoarthritis and Cartilage 16, 883-889.

Rutherford, D.J., Hubley-Kozey, C.L., Stanish, W.D., 2010. The neuromuscular demands of altering foot progression angle during gait in asymptomatic individuals and those with knee osteoarthritis. Osteoarthritis and Cartilage 18, 654-661.

Seth, A., Dong, M., Ricardo, M., Delp, S., 2019. Muscle Contributions to Upper-Extremity Movement and Work from a Musculoskeletal Model of the Human Shoulder. Frontiers of Neurorobotics 13:90.

Shkuratova, N., Morris, M.E., Huxham, F., 2004. Effects of age on balance control during walking. Archives of Physical Medicine and Rehabilitation 85(4), 582-588.

Shull, P.B., Shultz, R., Silder, A., Dragoo, J.L., Besier, T.F., Cutkosky, M.R., Delp, S.L., 2013. Toe-in gait reduces the first peak knee adduction moment in patients with medial compartment knee osteoarthritis. Journal of Biomechanics 46, 122-128.

Simic, M., Hinman, R., Wrigley, T., Bennell, K., Hunt, M., 2011. Gait modification strategies for altering medial knee joint load: a systematic review. Arthritis Care \& Research 63, 405426.

Tateuchi, H., Koyama, Y., Akiyama, H., Goto, K., So, K., Kuroda, Y., Ichihashi, N., 2017. Daily cumulative hip moment is associated with radiographic progression of secondary hip osteoarthritis. Osteoarthritis and Cartilage 25, 1291-1298.

Thorp, L.E., Sumner, D.R., Wimmer, M.A., Block, J.A., 2007. Relationship between pain and medial knee joint loading in mild radiographic knee osteoarthritis. Arthritis Care \& Research 57, 1254-1260.

Uhlrich, S.D., Silder, A., Beaupre, G.A., Shull, P.B., Delp, S.L., 2018. Subject-specific toe-in or toe-out gait modifications reduce the larger knee adduction moment peak more than a nonpersonalized approach. Journal of Biomechanics 66, 103-110.

Uhlrich, S.D, Kolesar, J.A., Kidzinski, L., Boswell, M.A., Silder, A., Gold, G.E., Delp, S.L., Beaupre, G.S., 2020. Personalization improves the biomechanical efficacy of foot progression angle modifications in individuals with medial knee osteoarthritis. medRxiv

Wesseling, M., Groote, F. de, Meyer, C., Corten, K., Simon, J.P., Desloovere, K., Jonkers, I., 2015. Gait alterations to effectively reduce hip contact forces. Journal of Orthopaedic Research 33, 1094-1102.

Xia, H., Charlton, J.M., Shull, P.B., Hunt, M.A., 2020. Portable, automated foot progression angle gait modification via a proof-of-concept haptic feedback-sensorized shoe. Journal of Biomechanics 107, 109789 
medRxiv preprint doi: https://doi.org/10.1101/2022.01.10.22268858; this version posted January 11, 2022. The copyright holder for this preprint (which was not certified by peer review) is the author/funder, who has granted medRxiv a license to display the preprint in perpetuity.

It is made available under a CC-BY-ND 4.0 International license .

TABLES \& FIGURES

\begin{tabular}{|lc|}
\hline Characteristic $(\mathbf{N}=\mathbf{5 0})$ & Mean $($ SD) \\
\hline \hline Age $(\mathrm{yr})$ & $61.34(7.81)$ \\
\hline Height $(\mathrm{m})$ & $1.72(0.11)$ \\
\hline Mass $(\mathrm{kg})$ & $76.24(15.43)$ \\
\hline BMI $\left(\mathrm{kg} / \mathrm{m}^{\wedge} 2\right)$ & $25.77(3.83)$ \\
\hline Gender & M: 31 \\
& F: 19 \\
\hline Static Knee Angle $\left({ }^{\circ}\right):$ & Ipsilateral: $-2.38(3.56)$ \\
Varus $(-)$ Valgus $(+)$ & Contralateral: $-1.02(3.58)$ \\
\hline $\begin{array}{l}\text { Participants who reduced peak KAM with a } \\
\text { modified FPA }\end{array}$ & Peak 1 only: $\mathrm{n}=11$ \\
& Peak 2 only: $\mathrm{n}=14$ \\
\hline Gait Speed (m/s) & Peak 1 \& Peak 2: $\mathrm{n}=25$ \\
\hline Kellgren and Lawrence Grade & $1.24(0.12)$ \\
\hline $\begin{array}{l}\text { Medial Knee Pain: } \\
0 \text { (no pain) - 10 (worst pain) }\end{array}$ & $\square: 12, \square: 27, \square: 11$ \\
\hline
\end{tabular}

Table 1: Demographics of the fifty participants with symptomatic medial knee osteoarthritis included in the analysis. Standard deviation (SD) is reported in parentheses. 
medRxiv preprint doi: https://doi.org/10.1101/2022.01.10.22268858; this version posted January 11, 2022. The copyright holder for this preprint (which was not certified by peer review) is the author/funder, who has granted medRxiv a license to display the preprint in perpetuity.

It is made available under a CC-BY-ND 4.0 International license.

\begin{tabular}{|l|cc|ccc|ccc|}
\hline \multirow{2}{*}{ Parameter } & \multicolumn{2}{|c|}{ Baseline } & \multicolumn{3}{c|}{$\mathbf{1 0}^{\circ}$ Toe-in } & \multicolumn{3}{c|}{ 10 $^{\circ}$ Toe-out } \\
& mean & SD & mean & SD & p-value & mean & SD & p-value \\
\hline \hline FPA $\left(^{\circ}\right)$ & 6.88 & 3.46 & -2.79 & 3.49 & $<\mathbf{0 . 0 0 1}$ & 16.53 & 3.41 & $<\mathbf{0 . 0 0 1}$ \\
Stride Length (m) & 1.33 & 0.14 & 1.29 & 0.13 & $<\mathbf{0 . 0 0 1}$ & 1.29 & 0.13 & $<\mathbf{0 . 0 0 1}$ \\
Step Width (m) & 0.18 & 0.04 & 0.19 & 0.04 & 0.8629 & 0.22 & 0.05 & $<\mathbf{0 . 0 0 1}$ \\
1st Peak KAM $(\% \mathrm{BW} / \mathrm{H})$ & 3.69 & 1.17 & 3.41 & 1.11 & & 3.80 & 1.23 & \\
2nd Peak KAM $(\% \mathrm{BW} / \mathrm{H})$ & 2.72 & 0.98 & 2.72 & 1.01 & & 2.42 & 1.06 \\
\hline
\end{tabular}

Table 2: Spatiotemporal parameters and KAM outcome measures for participants during baseline gait, $10^{\circ}$ toe-in and $10^{\circ}$ toe-out. Note, p-values are not reported for the peak KAM values since participants were selected based on their ability to reduce their peak KAM. 


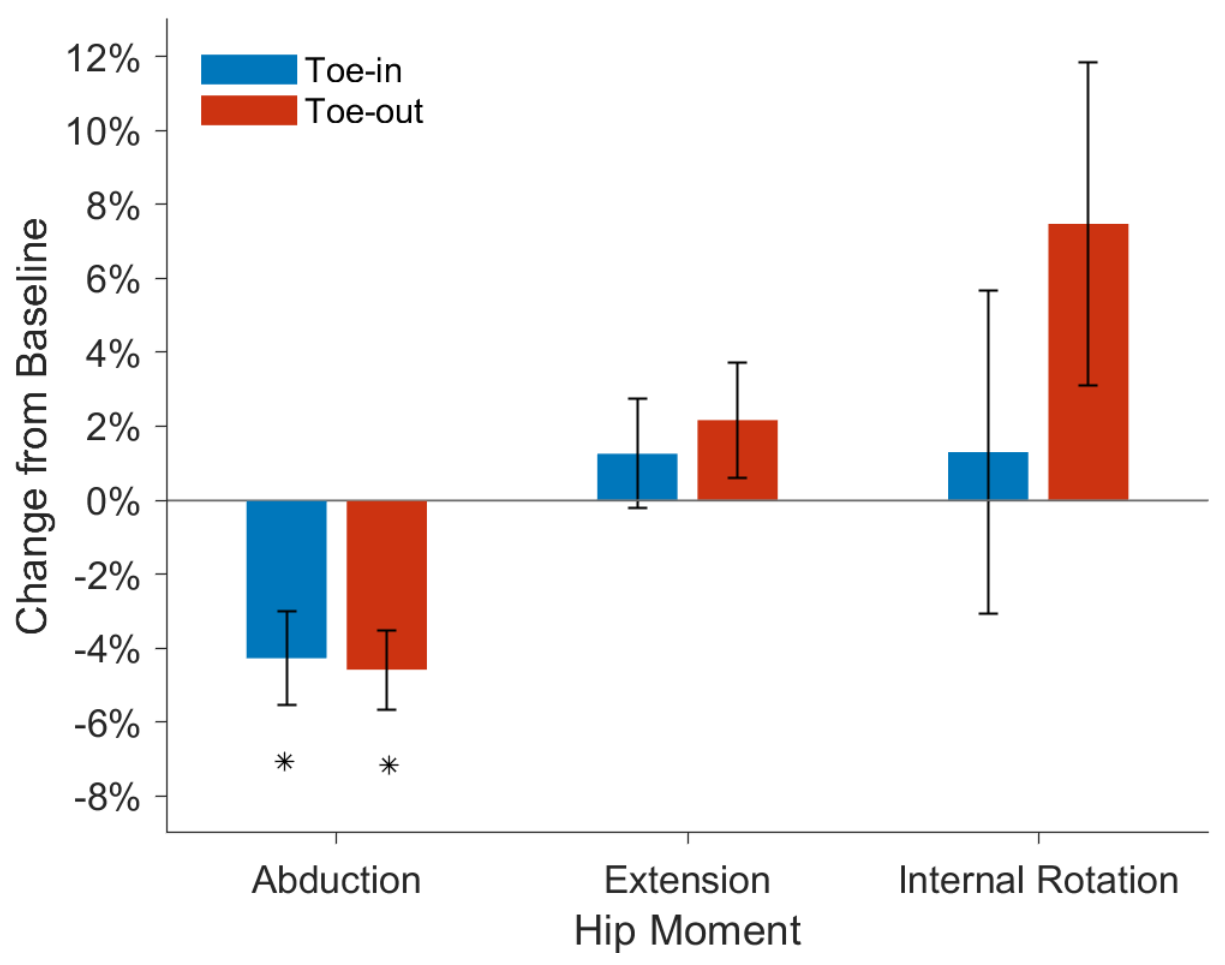

Figure 1: Participants reduced their early-stance hip abduction moment for $10^{\circ}$ toe-in (blue) and $10^{\circ}$ toe-out (red) gait as compared to baseline. No significant differences were observed between baseline gait and toe-in or toe-out gait for the early-stance hip extension and internal rotation moment. $\left({ }^{*} \mathrm{p}<0.005\right)$ 

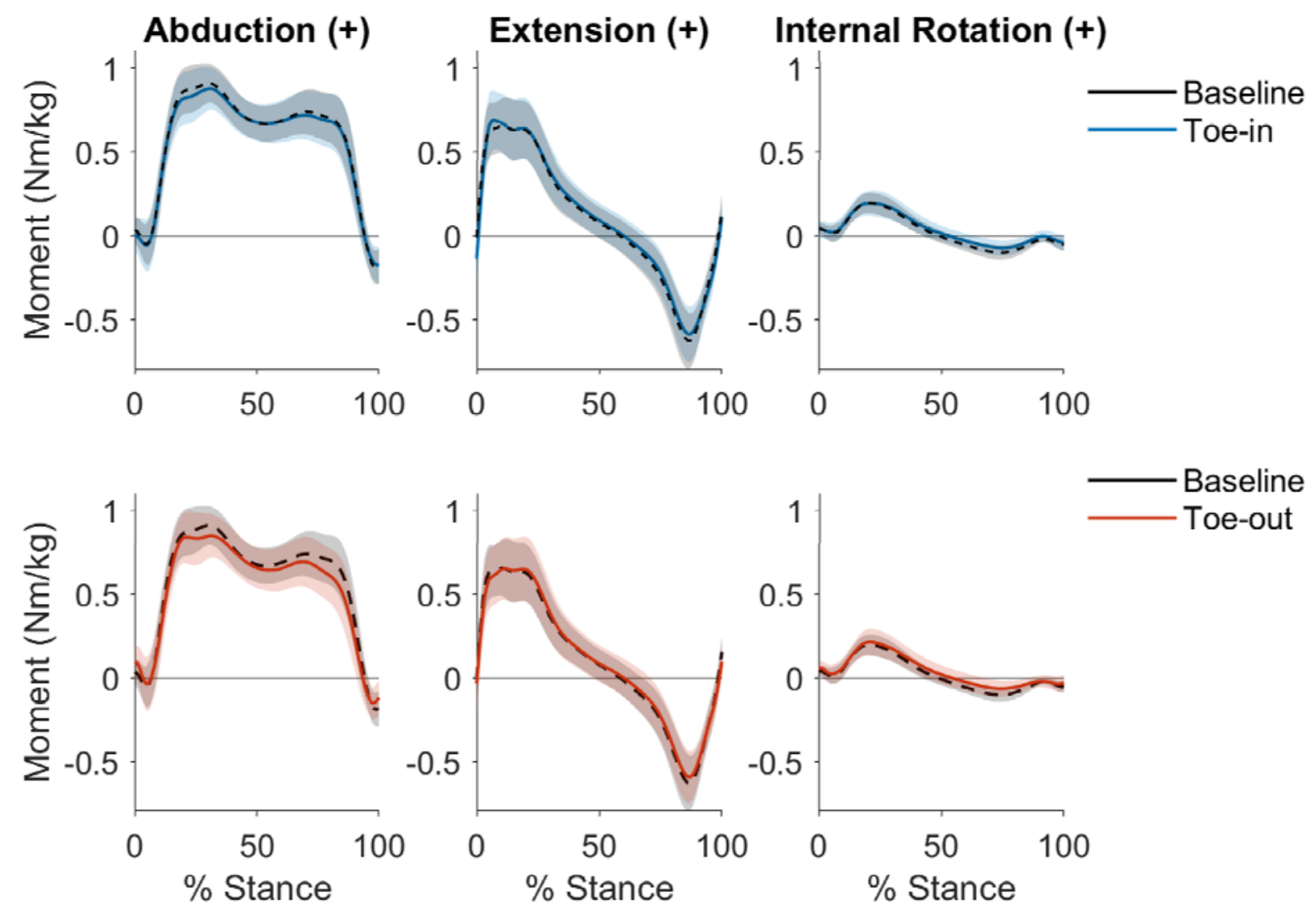

Figure 2: Ensemble-averaged hip moment curves, normalized by bodyweight, for all participants $(\mathrm{n}=50)$ during stance phase. Baseline gait (black, dashed) hip moments are compared to $10^{\circ}$ toe-in gait (blue, solid) in the first row and $10^{\circ}$ toe-out gait (red, solid) in the second row. The shaded region indicates one standard deviation from the average moment. 


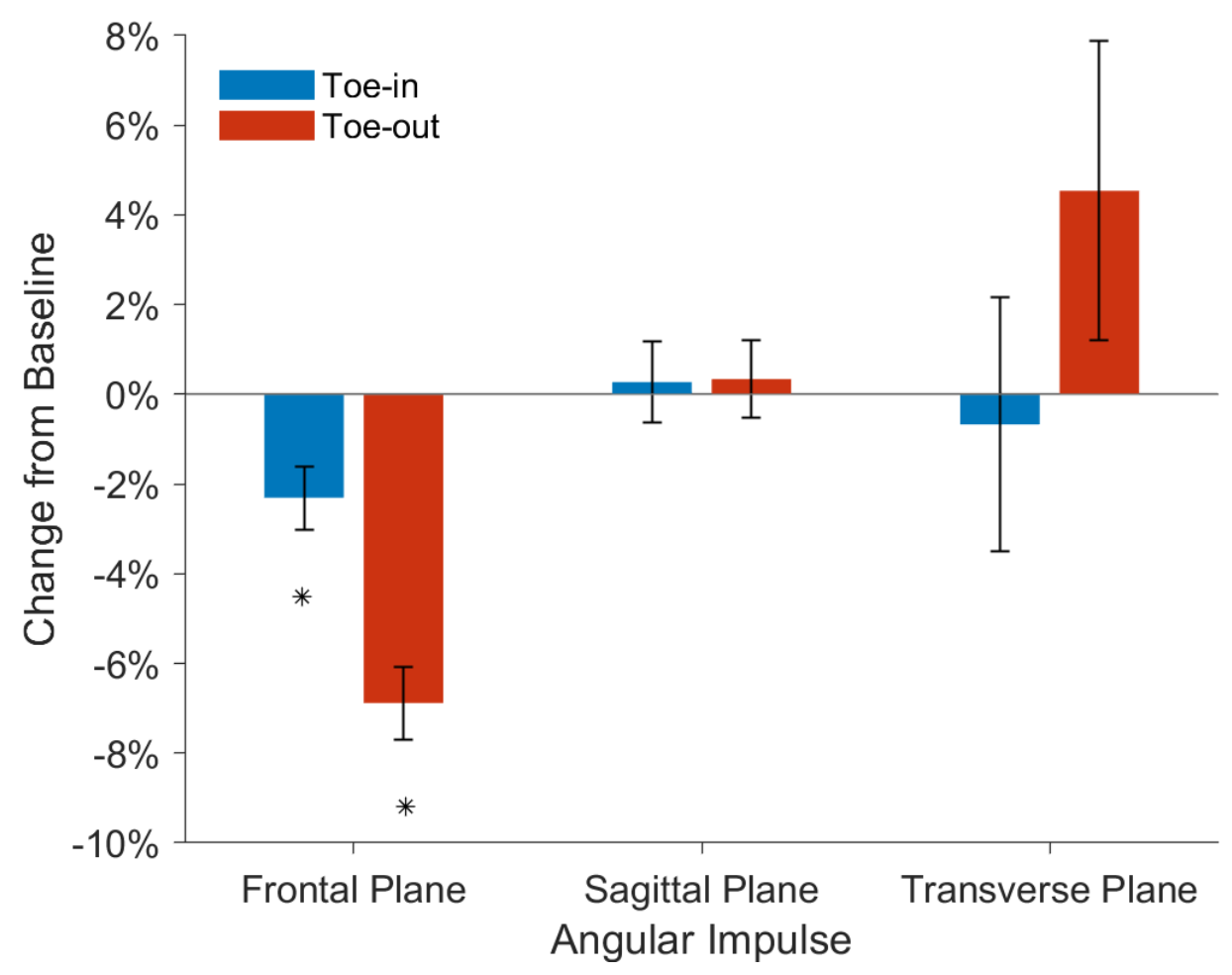

Figure 3: Participants reduced their frontal plane angular impulse during $10^{\circ}$ toe-in (blue) and $10^{\circ}$ toe-out (red) gait compared to baseline. No significant differences were observed between baseline and toe-in or toe-out gait for the angular impulse in the sagittal and transverse plane. $(* \mathrm{p}<0.01)$ 

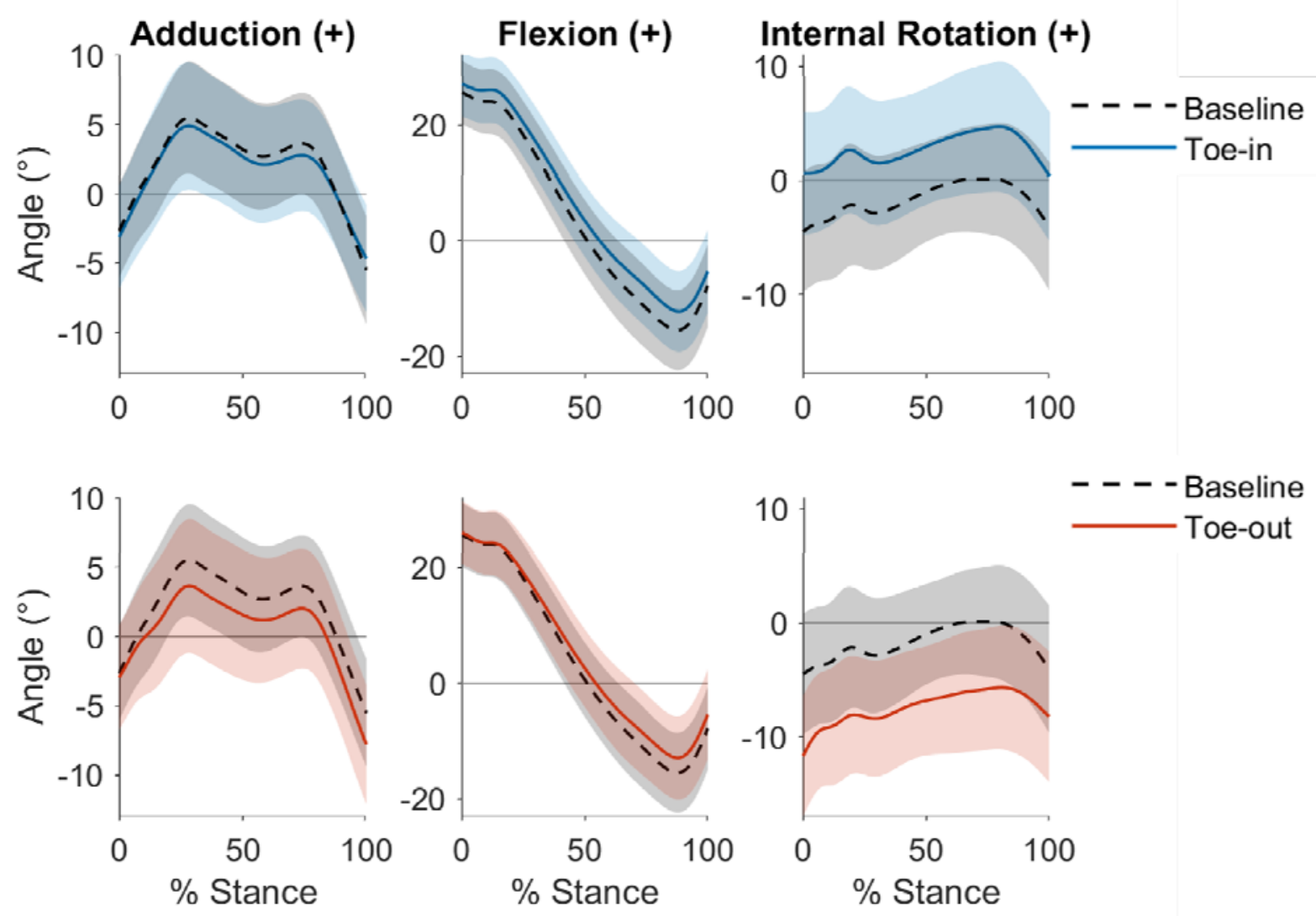

Figure 4: Ensemble-averaged hip kinematics for all participants $(n=50)$ during stance phase.

Baseline gait (black, dashed) hip angles are compared to $10^{\circ}$ toe-in gait (blue, solid) in the first row and $10^{\circ}$ toe-out gait (red, solid) in the second row. The shaded region indicates one standard deviation from the average hip angle. The peak hip adduction angle and the peak hip extension angle are significantly reduced with $10^{\circ}$ toe-in gait and $10^{\circ}$ toe-out gait compared to baseline $(\mathrm{p}<0.01)$. The peak hip internal rotation angle is significantly increased for $10^{\circ}$ toe-in gait and reduced for $10^{\circ}$ toe-out gait compared to baseline $(\mathrm{p}<0.001)$. 